\title{
Statistical Analysis of Common Diseases of College Students According to Different Living Areas of Cities, Towns and Rural Areas
}

\author{
Hongjun Teng ${ }^{1, a^{*}}$, Zhenbo Bao ${ }^{2, ~ b}$, Hongying Sun ${ }^{2, c}$ and Yu Shi ${ }^{2, d}$ \\ ${ }^{1}$ Clinic, Tianjin Agricultural University, Tianjin, China, 300384 \\ ${ }^{2}$ Engineering and Technology College, Tianjin Agricultural University, Tianjin, China, 300384 \\ a*hongjun-teng@163.com, bbaozhenbo@sohu.com, csunn@tjau.edu.cn, dsyych83@tjau.edu.cn
}

Keywords: College students; Common diseases; Different Living area; Statistical analysis

\begin{abstract}
In order to understand the types and distribution characteristics of the common diseases in Tianjin Agricultural College, to provide a basis for medical and health care, the clinical data of college students from September 2016 to December 2016 in Tianjin Agricultural College Hospital were analyzed statistically. The conclusions were drawn: colds, upper respiratory tract infections, bronchitis, excessive internal heat, sprain, gastroenteritis, skin irritation, toothache, stomach pain, eye fatigue, conjunctivitis, dysmenorrheal, rhinitis, otitis media and beriberi were the common diseases of college students, and the top 6 of the common diseases were colds, upper respiratory tract infection, excessive internal heat, sprain, gastroenteritis and skin allergies. According to the different living areas, the conclusions were also obtained: the college students from rural areas with a high incidence rate of cold, upper respiratory tract infections, excessive internal heat and gastroenteritis; the college students from the city with a high incidence rate of skin irritation; and the incidence rate of sprain had no obvious relationship with the living areas. According to different students, health care workers in colleges and universities should carry out medical and health education, improve the resistance of college student's disease, and reduce the incidence of common diseases of college students.
\end{abstract}

\section{Introduction}

College students are the important resources of scientific and technological progress and social development, and are charged with the task of building the country. The health of college students has a far-reaching impact on society, has become the focus of the whole society [1-3]. Ordinary college students are generally 17-25 years of age, the physiological function and body function gradually mature, psychological adaptability continues to mature and physical life in a higher level, are healthy groups with less incidence of various diseases. However, due to the lack of health knowledge, in a relatively independent life, affected by some unhealthy lifestyles and emotional factors such as psychological regulation out of control, easily lead to the occurrence of certain diseases and accidental injury [4-6]. In China's colleges and universities, students each year due to a variety of diseases led to the suspension of the situation gradually increased [7-8]. To promote the healthy growth of students is the starting point and the foothold of all the work of the school, and constantly improve the physical health of college students is an important task of higher education. I have been engaged in comprehensive out-patient diagnosis and treatment, health care, health education more than 10 years, and profoundly realize that through health education, so that students can master the knowledge of diseases prevention and reduce accidental injury, which is a strong guarantee for healthy life of college students and successfully complete their studies. The clinical data of college students from September 2016 to December 2016 in Tianjin Agricultural College Hospital were analyzed statistically, the results of statistical analysis can provide basis for understanding the distribution of common diseases of college students, the characteristics and the main causes of disease, and carrying out preventive health and health education and other work. 


\section{Statistical Analysis of Common Diseases of College Students}

In the outpatient treatment work, the clinical data of college students from September 2016 to December 2016 in Tianjin Agricultural College hospital were analyzed statistically, a total of 669 people for the analysis of the object. The return visits were not within the scope of statistics, of which 263 were male students, 406 female students, in the age of 17-25 years. The 15 kinds of common diseases of college students are colds, upper respiratory tract infections, bronchitis, excessive internal heat, sprain, gastroenteritis, skin irritation, toothache, stomach pain, eye fatigue, conjunctivitis, dysmenorrheal, rhinitis, otitis media and beriberi, the distribution of the number and percentage as shown in Table 1.

Table 1 Distribution of numbers and percentage of 15 kinds of common diseases of college students

\begin{tabular}{|c|c|c|c|}
\hline No. & Name of common disease & Numbers & Proportion \\
\hline 1 & Colds & 213 & $31.84 \%$ \\
\hline 2 & Upper respiratory tract infections & 91 & $13.60 \%$ \\
\hline 3 & Excessive internal heat & 78 & $11.66 \%$ \\
\hline 4 & Sprain & 45 & $6.73 \%$ \\
\hline 5 & Gastroenteritis & 43 & $6.43 \%$ \\
\hline 6 & Skin irritation & 40 & $5.98 \%$ \\
\hline 7 & Toothache & 38 & $5.68 \%$ \\
\hline 8 & Stomach pain & 25 & $3.74 \%$ \\
\hline 9 & Bronchitis & 23 & $3.44 \%$ \\
\hline 10 & Eye fatigue & 20 & $2.99 \%$ \\
\hline 11 & Conjunctivitis & 15 & $2.24 \%$ \\
\hline 12 & Dysmenorrheal & 10 & $1.64 \%$ \\
\hline 13 & Rhinitis & 9 & $1.49 \%$ \\
\hline 14 & Otitis media & 8 & $1.35 \%$ \\
\hline 15 & Beriberi & & $1.20 \%$ \\
\hline
\end{tabular}

Among the common diseases, the proportion of colds, upper respiratory tract infections and excessive internal heat were $31.84 \%, 13.60 \%$ and $11.66 \%$ respectively, which was caused by the cold season and dry climate, in addition, students eat less vegetables and fruits.

\section{Statistical Analysis of 6 Kinds of Common Diseases according to Different Living Areas of Cities, Towns and Rural Areas}

The higher distribution ratio of 6 kinds of common diseases were colds, upper respiratory tract infection, excessive internal heat, sprain, gastroenteritis and skin allergies, the proportion of distribution of the 6 kinds of common diseases are shown with the bar chart in Figure 1 to Figure 6. As shown in Figure 1 to Figure 6, according to the different living areas of cities, towns and rural areas, the following conclusions were obtained: the college students from rural areas with a high incidence rate of cold $(47.52 \%)$, upper respiratory tract infections $(50.82 \%)$, excessive internal heat (50\%), gastroenteritis $(42.86 \%)$; the college students from the city with a high incidence rate of skin irritation $(48.15 \%)$; and the incidence rate of sprain had no obvious relationship with the living $\operatorname{areas}(32.26 \%, 32.26 \%, 35.48 \%)$. The reasons for the statistical conclusions: most of the rural students because of family conditions are more difficult, congenital deficiencies and eating dystrophy, leading to immune dysfunction, coupled with improper care, poor health awareness, so susceptible to a variety of diseases. However, students from the city less sun and wind, the function of skin adapting to the changes of the environment and temperature is poor, so prone to skin allergies. 


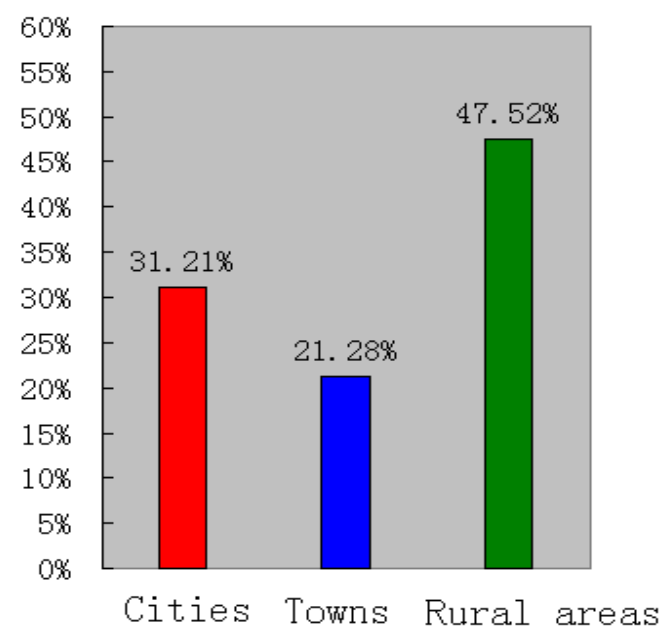

Figure 1. The distribution proportion of cold according to different living areas of cities, towns and rural areas

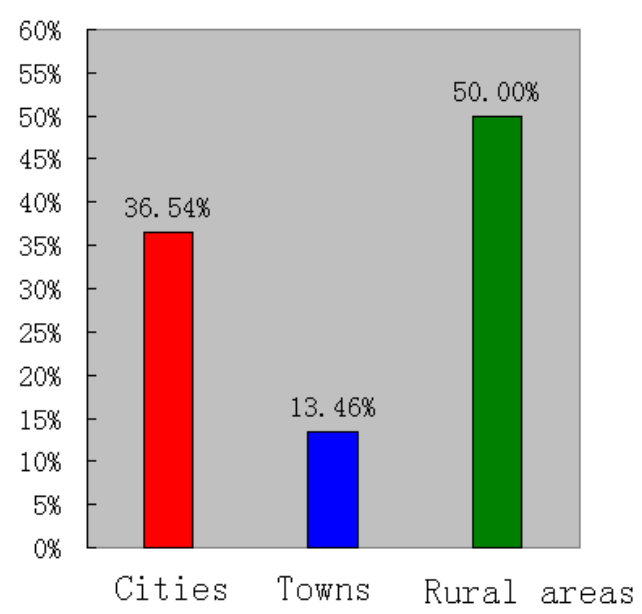

Figure 3. The distribution proportion of excessive internal heat according to different living areas of cities, towns and rural areas

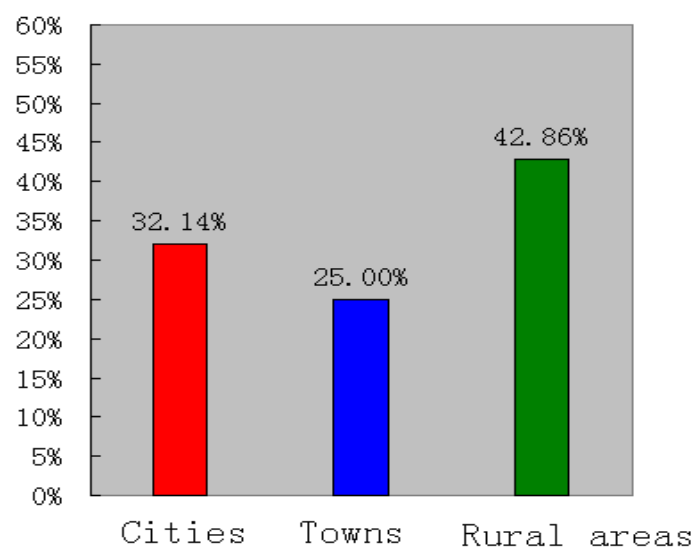

Figure 5. The distribution proportion of gastroenteritis according to different living areas of cities, towns and rural areas

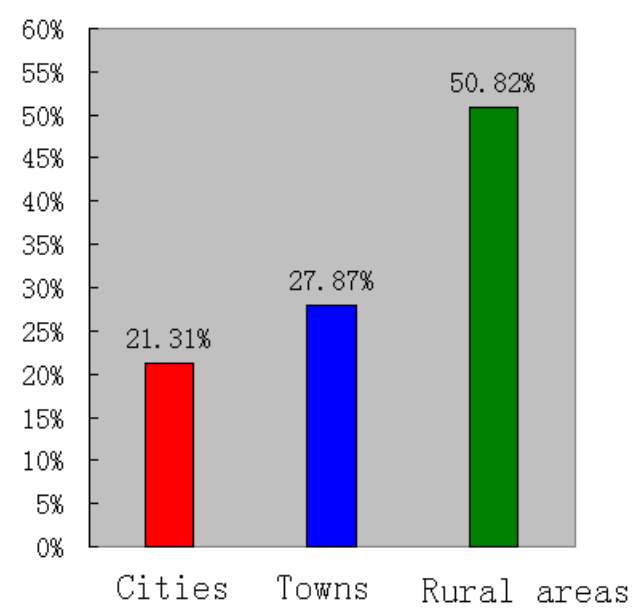

Figure 2. The distribution proportion of upper respiratory tract infection according to different living areas of cities, towns and rural areas

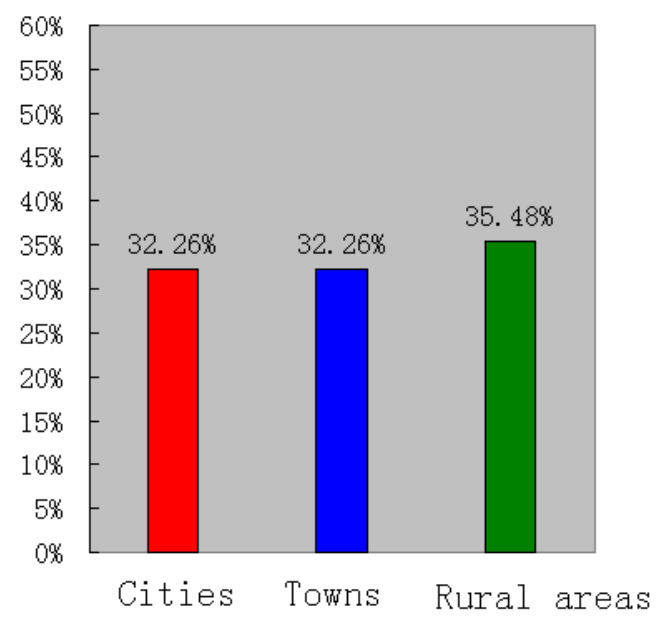

Figure 4. The distribution proportion of sprain according to different living areas of cities, towns and rural areas

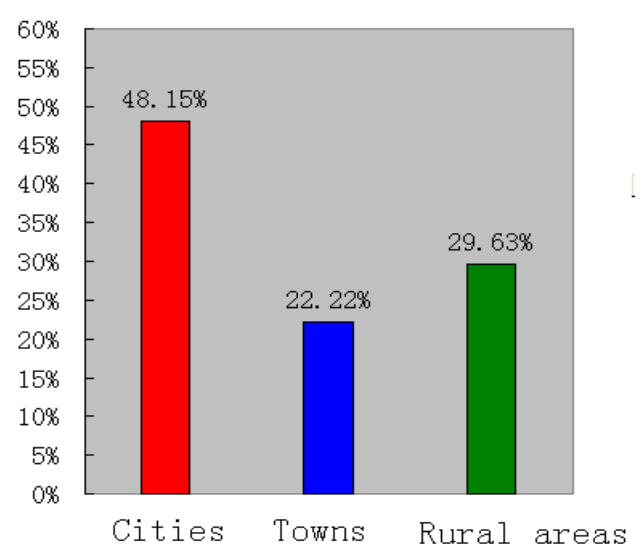

Figure 6. The distribution proportion of skin allergies according to different living areas of cities, towns and rural areas 


\section{Summary}

Through the statistical analysis of the outpatient common diseases of college students in autumn and winter festival of Tianjin Agricultural College, it was found that 15 kinds of colds, upper respiratory tract infections, bronchitis, excessive internal heat, sprain, gastroenteritis, skin irritation, toothache, stomach pain, eye fatigue, conjunctivitis, dysmenorrheal, rhinitis, otitis media and beriberi are the common diseases of college students, and the proportion of colds, upper respiratory tract infections and bronchitis was high. Rural students due to economic conditions, single diet and other reasons, the incidence rates of colds, upper respiratory tract infection, excessive internal heat and gastroenteritis were high, and from the city's college students due to poor natural climate adaptation, the incidence rate of skin allergies was high, and the incidence rate of sprain had no obvious relationship with the living areas. It is suggested that health care workers should adopt appropriate preventive health care and health education measures to improve the health level of college students according to the regularity, characteristics and causes of autumn and winter seasons of college students [9-10].

\section{Acknowledgements}

This work was financially supported by the Project of Tianjin Agricultural University Education and Teaching Reform Research (2016-A-04).

\section{References}

[1] W.Y. Yan, X.M. Jia: For all Health, Vol. 10 (2016) No.15, p.8.

[2] Q.B. Wang, X. Shi and Y.Y. Wei: China Medical Herald, Vol. 6 (2009) No.26, p.120.

[3] X.C. Sun: China Medical Engineering, Vol. 19 (2011) No.12, p.149-150.

[4] W.J. Liu: Journal of Guangdong University of Petrochemical Technology, Vol. 23 (2013) No.5, p.35-37.

[5] Z, H, Lu: Journal of Qiqihar University of Medical, Vol. 36 (2015) No.11, p.1664-1665.

[6] S.F. Wang, L.Z. Xu and J. Fang: Chin J Sch Health, Vol. 28 (2007) No.7, p.609-610.

[7] P.H. Wang: Journal of Anhui Institute of Education, Vol. 25 (2007) No.6, p.89-92.

[8] Y.H. Feng, M.Y. Wu and H.L. Chen: Journal Traditional Medicine Management, Vol. 24 (2016)

[9] No.10, p.167-169.

[10] S.M. Sun: Chin J Sch Health, Vol. 35 (2014) No.11, p.1739-1741.

[11]Q.T. Wu, J.W. Gan: Health Medicine Research and Practice, Vol. 12 (2015) No.5, p.86-88. 